\title{
Why we need more complex gene diagnostic: the case study of exome from patient with congenital glaucoma
}

\author{
Dinara Ivanoshchuk \\ ICG SB RAS, Novosibirsk, Russia \\ dinara@bionet.nsc.ru
}

\author{
Mikhail Voevoda \\ ICG SB RAS, Novosibirsk, Russia \\ voevoda@bionet.nsc.ru \\ Konstantin Gunbin \\ ICG SB RAS, Novosibirsk, Russia \\ NSU, Novosibirsk, Russia \\ genkvg@bionet.nsc.ru
}

\author{
Natalia Konovalova \\ TSMA, Tumen, Russia \\ doctork@bk.ru
}

\begin{abstract}
Usually, a detailed analysis of well-known diseases-related genes for the presence of pathogenic variants does not give any meaningful result, despite the good agreement between the patient's diagnosis and the available data on the mutations manifestation in these genes. This study, we try to disentangle such a cases using gene network aware exome analysis and variants prioritization.
\end{abstract}

Keywords - gene network based mutation prioritization, exome analysis, glaucoma

\section{MOTIVATION AND AIM}

One of the major challenge in the interpretation of exomewide results is the absence of any information about the interaction of weakly damaging mutations (likely-benign and or benign) and mutations with an unknown effect in the compounds. Earlier huge efforts directed towards disentangling biological reasons of single nucleotide variations expression and penetrance of genes implicated in complex diseases determination were taken $[1,2]$. Most of approaches intended to solve this problem are based on the analysis of topologies gene network of trait and/or on the data on gene-gene or protein-protein functional interaction. This study, we try to disentangle such a type of cases using our own simplified gene network aware exome analysis and variants prioritization. To do so, we used data from anonymous patient with healthy parents and evidences of congenital glaucoma.

\section{METHODS}

Paired reads exome library of anonymized patient with signatures of congenital glaucoma was mapped onto the reference genome using bowtie2-align-s. SNVs were called using Platypus v. 0.8.1 software, we used all default filtering options, except one considering duplicated reads that we switched in two alternative states. As a result, two VCF files were obtained, containing SNVs detected based on all mapped reads and mapped reads without duplicated ones. Next, SNVs from these two VCF files were additionally filtered taking into account the quality of the nucleotides confirming them. First, all SNVs marked by Platypus with the badReads label were disregarded; these are SNVs supported by reads from only one DNA strand having low base calling quality close to the SNV (Phred quality <20). Second, using the GATK v. 4.1.4.1 software, we selected out all SNVs that did not satisfy the lowest local coverage threshold. This work, we did not take into account deletion / insertion variants (INDELs), because we identified that overwhelming number of INDELs (>96\%) was marked with the alleleBias label by the Platypus; this label indicates that the allele frequency is statistically significantly lower than expected in the heterozygous state. At the final, we got two cleaned VCF files containing SNVs, supported by all mapped reads and mapped reads without duplicated ones.

The functional annotation of known missense SNVs and SNVs located in known splice sites was done using dbNSFP v. 4.0a and GnomAD v. 2.1.1 databases. In-house Perl scripts were implemented for variants extraction using Tabix indexing technology. Information on SNV functional significance was taken in the form of 40 normalized rank scores from the dbNSFP database. For each SNV, the maximum occurrence frequency was taken from the GnomAD database.

After SNVs annotating, we prioritized the genes containing these SNVs. For the constructing gene scoring formula for gene prioritization, we used both previously obtained variables for individual SNVs (dbNSFP boot median and GnomAD_max freq), and the gene-wise characteristics, indicating functional significance of the gene. We used the following gene-wise characteristics: 1) the pLI score, the probability of being loss-of-function intolerant (the pLI_value), 2) the average occurrence frequency of de novo mutations in the gene from the mirDNMR database (the DNMR); 3 ) the sum of the maximum length of the protein sequence and the number of exons in the gene (the AA_SS_Length).

This work, we additionally take into consideration the GADO score [2] reflecting the gene significance in the gene network relating to glaucoma phenotype (the GADO value) and the the normalized level of the gene expression in the affected tissues - cornea, iris and anterior chamber of the eye (the TISS_value).

The final simple gene scoring formula was as follows: $\mathrm{S}(\mathrm{g})=$ TISS_value*GADO_value*pLI_value*DNMR_coeff $\left.* \sum\{\text { dbNSFP boot_median*(1-GnomAD_max_freq })^{*} \mathrm{AC}\right\}$, where $\mathrm{AC}$ is the number of alternative alleles at the SNV locus (1 - heterozygote; 2 - homozygote), $\sum$ is a sum across the SNV loci containing alternative variants in the gene, DNMR_coeff $=\log 10\{$ AA_SS_Length/DNMR $\}$.

\section{RESULTS}

A simple and effective guideline for searching and prioritizing genes and exome SNVs associated with a risk to diseases was developed. Using this pipeline, we were able to uncover small number of mutated protein-coding genes 
involved in cornea and eye chamber functioning as well as in whole eye (Table 1). Despite very small number of genes involved in cornea and eye chamber functioning, this gene list significantly enriched with Visual phototransduction pathway in REACTOME $(\mathrm{p}=2.3 \mathrm{E}-05)$ and with basolateral cell membrane in GO CC $(\mathrm{p}=5.4 \mathrm{E}-04)$.

\section{ACKNOWLEDGMENT}

This work supported by the RFBR grant \# 18-315-00297.

\section{REFERENCES}

[1] Oulas A, et al. (2019) Selecting variants of unknown significance through network-based gene-association significantly improves risk prediction for disease-control cohorts. Sci Rep. 9(1):3266.

[2] Deelen P, et al. (2019) Improving the diagnostic yield of exomesequencing by predicting gene-phenotype associations using largescale gene expression analysis. Nat Commun.10(1):2837
Table 1. The list of all genes associated with glaucoma and related to cornea and eye chamber functioning as well as whole eye functioning

\begin{tabular}{|c|c|c|}
\hline $\begin{array}{r}\text { Mutated top } \\
\text { genes in whole } \\
\text { eye }\end{array}$ & $\begin{array}{l}\text { Mutated top genes } \\
\text { functioning in } \\
\text { cornea }\end{array}$ & $\begin{array}{l}\text { Mutated top genes } \\
\text { functioning in } \\
\text { eye chamber }\end{array}$ \\
\hline CFB & CFB & CFB \\
\hline GUCY2D & GUCY2D & GUCY2D \\
\hline ELAC2 & CNGB1 & CNGB1 \\
\hline CCDC170 & MYO7A & CNGB3 \\
\hline CNGB1 & BBS2 & \\
\hline MYO7A & CNGB3 & \\
\hline HNF1A & HNF1A & \\
\hline CNGB3 & DAG1 & \\
\hline BBS2 & & \\
\hline HEXB & & \\
\hline DAG1 & & \\
\hline
\end{tabular}

